\title{
Guest-Editorial
}

\section{Assembly structures under crowd dynamic excitation}

\author{
Aleksandar Pavic \\ Professor of Vibration Engineering and EPSRC Advances Research Fellow, University of Sheffield, UK \\ E-mail: a.pavic@sheffield.ac.uk
}

The dynamic performance of large assembly structures, such as sports stadia, concert halls and footbridges, under crowd-induced dynamic loading is currently recognised as one of the most important and still unresolved areas of concern for civil structural engineers worldwide. This is due to the disastrous consequences that would arise if the structural failure or panic caused by severe crowd-induced vibrations should ever occur. Arguably, the state of understanding of crowd dynamic loading today is comparable to that of wind engineering just before the infamous Tacoma Narrows bridge disaster in November 1940. The aim of this special issue is, therefore, to present original contributions and enhance the current state-of-the-art related to civil engineering structures under crowd dynamic excitation.

The main reason for this global concern is the lack of reliable and consistent design guidance for structures under crowd dynamic loading. This is caused by the poor current understanding of the problem, which in turn is a consequence of inadequate research in the area over the last few decades. Starting from first principles, every vibration engineering problem can be rationalised into three parts: vibration source (i.e. what is the dynamic loading), vibration path (i.e. what is the structural mass, stiffness and damping) and vibration response and its effects on the 'receiver' (typically, the receiver is humans, occupying the structure but could also be vibration sensitive equipment). This general approach also applies to assembly structures under crowd dynamic loading and currently there are serious unresolved problems with each of them.

Firstly, crowd-induced dynamic loads are not well understood, in particular the effects of synchronisation (or lack of it). Secondly, structures occupied by crowds, such as grandstands, are quite complex and difficult to model mathematically even when they are empty. Moreover, the effects of crowds present on those structures, in terms of mass, damping and stiffness, have been observed in practice as quite significant, but no reliable and widely accepted method for modelling of these currently exists. Finally, even if one is able to calculate reliably a vibration response based on well defined vibration source and transmission path, the acceptability of such vibration would be very difficult to determine for assembly structures. For example, what levels of vibration would be acceptable for passive and active people attending a noisy rock concert? It is quite hard to judge this at the moment.

Over the last decade, advances in measurement technologies have created new and very promising opportunities to tackle these problems from a combined experimental and analytical angle. This is the right way forward, which is slowly but surely being accepted around the world. For example, in the last five years in the UK, there was a marked shift towards combining modal testing of as-built full-scale grandstands and their mathematical modelling, typically performed using finite element analysis. This is now a common approach widely adopted by industry in the UK aimed at reducing considerable uncertainties when performing assessment of vibration performance of large grandstands under crowd-induced dynamic loading. Current UK design guidance is probably the only in the world recommending both testing and analysis of all new grandstand structures.

The papers that have made it into this special issue deal with the first two parts of the general vibration engineering problem. Yoshida and Fujino used video records and pattern recognition to investigate forces from and synchronisa- 
tion of crowds walking over a laterally swaying footbridge in Japan. This is, indeed, a technology which has a lot of future potential for investigating crowd forces and their synchronisation which are practically impossible to measure directly. Sim et al. offered a proposal for modelling of joint crowd-structure dynamic system which could be used to interpret the aforementioned changes in modal properties observed on real grandstands and other structures occupied by large crowds. Similarly, Zhou and Ji provided a theoretical background for the estimation of dynamic characteristics of a spring-mass-beam system, which can be used for simplified modelling of behaviour of beam-like models of structures with large number of people on them. Finally, analysing response-only vibration data from crowd-occupied structures is a notoriously difficult task. However, Peeters et al. propose an efficient and reliable way for operational modal analysis aimed at estimating the dynamic properties of an occupied stadium structure during a football game. For this they used unique real-life data from a number of football matches gathered via a remotely controlled vibration monitoring system developed by Dr Paul Reynolds and other members of my research group at the University of Sheffield (http://vibration.shef.ac.uk). The system was installed on a real grandstand in Bradford, UK for a year. These data have also been used in a rather interesting Round Robin challenge to estimate modal properties of an occupied grandstand. Results of this exercise were presented in February 2005 at the 23rd International Modal Analysis Conference in Orlando, USA. This is when the idea for this special issue was conceived and after two years of hard work the main deliverable of this idea is in your hands eagerly waiting to be read. 

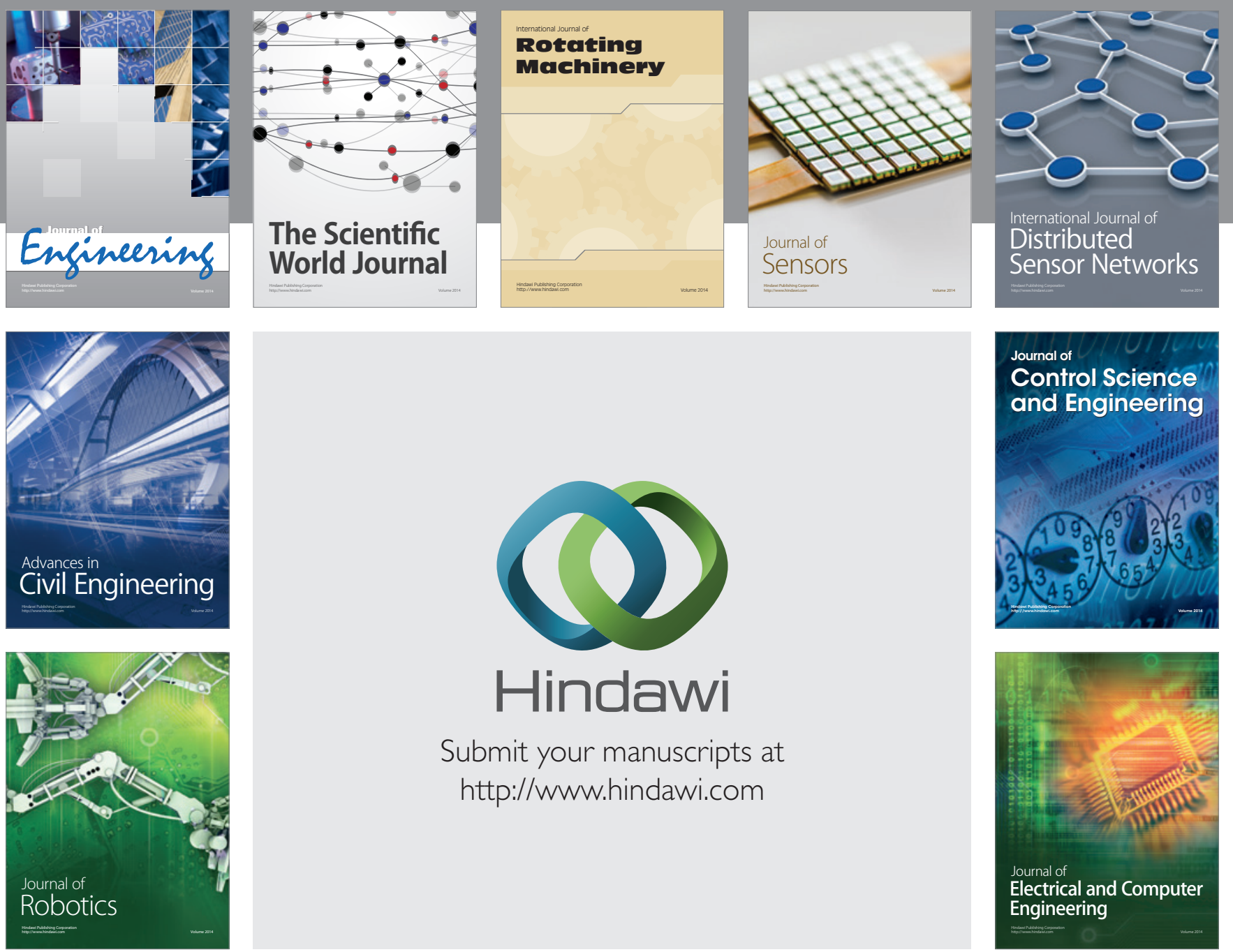

Submit your manuscripts at

http://www.hindawi.com
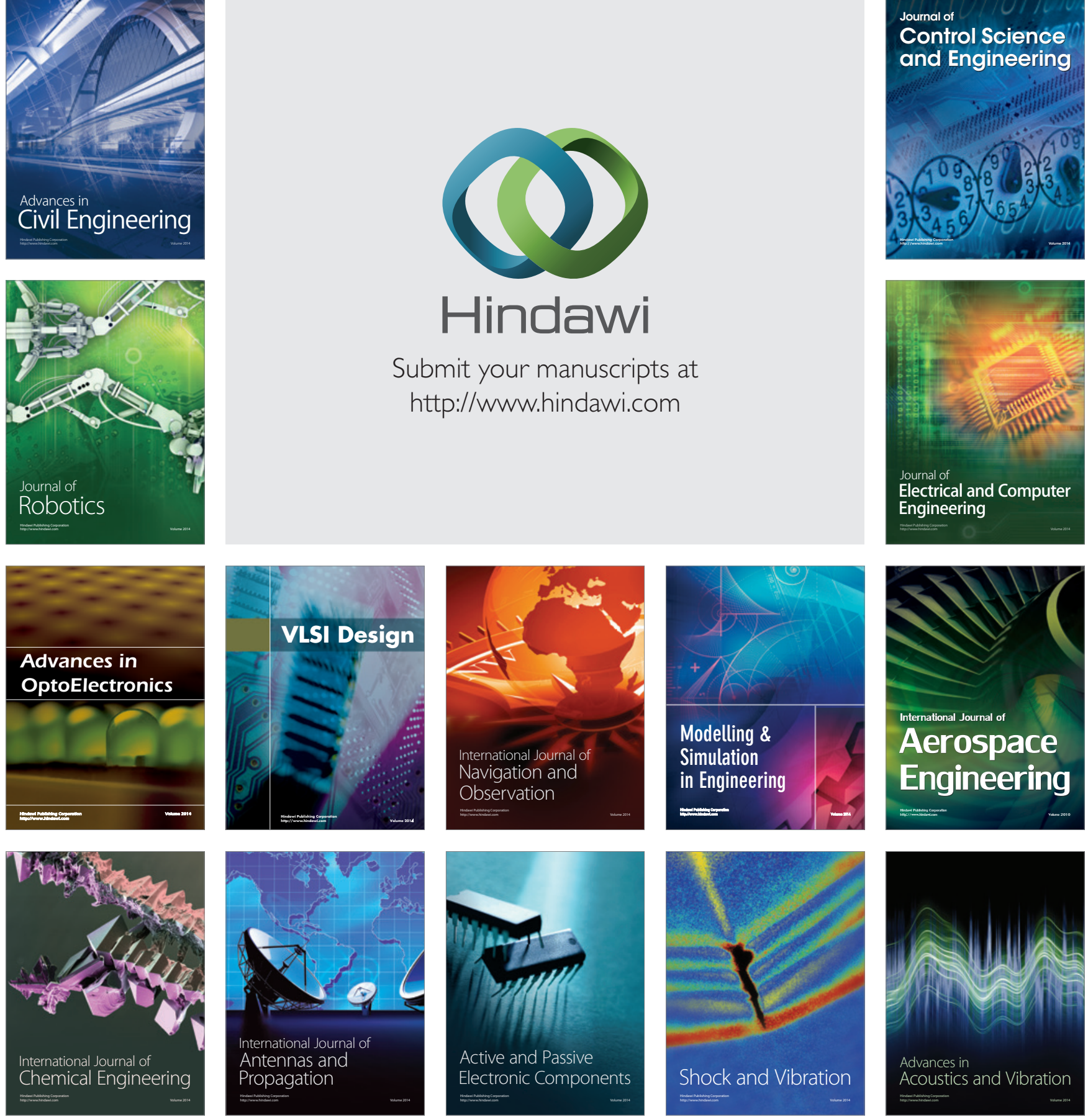